\title{
Composition theorems in communication complexity
}

\author{
Troy Lee ${ }^{1}$ and Shengyu Zhang ${ }^{2}$ \\ 1 Rutgers University, troyjlee@gmail.com \\ 2 The Chinese University of Hong Kong, syzhang@cse.cuhk.edu.hk
}

\begin{abstract}
A well-studied class of functions in communication complexity are composed functions of the form $\left(f \circ g^{n}\right)(x, y)=f\left(g\left(x^{1}, y^{1}\right), \ldots, g\left(x^{n}, y^{n}\right)\right)$. This is a rich family of functions which encompasses many of the important examples in the literature. It is thus of great interest to understand what properties of $f$ and $g$ affect the communication complexity of $\left(f \circ g^{n}\right)$, and in what way.

Recently, Sherstov [She09] and independently Shi-Zhu [SZ09b] developed conditions on the inner function $g$ which imply that the quantum communication complexity of $f \circ g^{n}$ is at least the approximate polynomial degree of $f$. We generalize both of these frameworks. We show that the pattern matrix framework of Sherstov works whenever the inner function $g$ is strongly balanced - we say that $g: X \times Y \rightarrow\{-1,+1\}$ is strongly balanced if all rows and columns in the matrix $M_{g}=[g(x, y)]_{x, y}$ sum to zero. This result strictly generalizes the pattern matrix framework of Sherstov [She09], which has been a very useful idea in a variety of settings [She08b,RS08,Cha07,LS09a,CA08,BHN09]. Shi-Zhu require that the inner function $g$ has small spectral discrepancy, a somewhat awkward condition to verify. We relax this to the usual notion of discrepancy.

We also enhance the framework of composed functions studied so far by considering functions $F(x, y)=f(g(x, y))$, where the range of $g$ is a group $G$. When $G$ is Abelian, the analogue of the strongly balanced condition becomes a simple group invariance property of $g$. We are able to formulate a general lower bound on $F$ whenever $g$ satisfies this property.
\end{abstract}

\section{Introduction}

Communication complexity studies the minimum amount of communication needed to compute a function whose input variables are distributed between two or more parties. Since the introduction by Yao [Yao79] of an elegant mathematical model to study this question, communication complexity has grown into a rich field both because of its inherent mathematical interest and also its application to many other models of computation. See the textbook of Kushilevitz and Nisan [KN97] for a comprehensive introduction to the field.

In analogy with traditional computational complexity classes, one can consider different models of communication complexity based on the resources available to the parties. Besides the standard deterministic model, of greatest interest to us will be a randomized version of communication complexity, where the parties have access to a source of randomness and are allowed to err with some small constant probability, and a quantum model where the parties share a quantum channel and the cost is measured in qubits.

Several major open questions in communication complexity ask about how different complexity measures relate to each other. The log rank conjecture, formulated by Lovász and Saks [LS88], asks if the deterministic communication complexity of a Boolean function $F: X \times Y \rightarrow\{0,1\}$ is upper bounded by a polynomial in the logarithm of the rank of the matrix $[F(x, y)]_{x, y}$. Another major open question is if randomized and quantum communication complexity are polynomially related for all total functions. We should mention here that the assumption of the function being total is crucial as an exponential separation is known for a partial function [Raz99]. 
One approach to these questions has been to study them for restricted classes of functions. Many functions of interest are block composed functions. For finite sets $X, Y$, and $E$, a function $f: E^{n} \rightarrow\{-1,+1\}$, and a function $g: X \times Y \rightarrow E$, the block composition of $f$ and $g$ is the function $f \circ g^{n}: X^{n} \times Y^{n} \rightarrow\{-1,+1\}$ defined by $\left(f \circ g^{n}\right)(x, y)=f\left(g\left(x^{1}, y^{1}\right), \ldots, g\left(x^{n}, y^{n}\right)\right)$ where $\left(x^{i}, y^{i}\right) \in X \times Y$ for all $i=1, \ldots, n$. For example, if $E=\{-1,+1\}$, the inner product function results when $f$ is PARITY and $g$ is AND, set-intersection when $f$ is OR and $g$ is AND, and the equality function when $f$ is AND and $g$ is the function IS-EQUAL, which is one if and only if $x=y$.

In a seminal paper, Razborov [Raz03] gave tight bounds for the bounded-error quantum communication complexity of block composed functions where the outer function $f$ is symmetric and the inner function $g$ is bitwise AND. In particular, this result showed that randomized and quantum communication complexity are polynomially related for such functions.

More recently, very nice frameworks have been developed by Sherstov [She07,She09] and independently by Shi and Zhu [SZ09b] to bound the quantum complexity of block composed functions that goes beyond the case of symmetric $f$ to work for any $f$ provided the inner function $g$ satisfies certain technical conditions. When $g$ satisfies these conditions, this framework allows one to lower bound the quantum communication complexity of $f \circ g^{n}$ in terms of the approximate polynomial degree of $f$, a classically well-studied measure. Shi and Zhu are able to get a bound on $f \circ g^{n}$ in terms of the approximate degree of $f$ whenever $g$ is sufficiently "hard" - unfortunately, the hardness condition they need is in terms of "spectral discrepancy," a quantity which is somewhat difficult to bound, and their bound requires that $g$ is a function on at least $\Omega(\log (n / d))$ bits, where $d$ is the approximate polynomial degree of $f$. Because of this, Shi-Zhu are only able to reproduce Razborov's results with a polynomially weaker bound.

Sherstov developed so-called pattern matrices which are the matrix representation of a block composed function when $g$ is a fixed function of a particularly nice form. Namely, in a pattern matrix the inner function $g:\{-1,+1\}^{k} \times([k] \times\{-1,+1\}) \rightarrow\{-1,+1\}$ is parameterized by a positive integer $k$ and defined by $g(x,(i, b))=x_{i} \cdot b$, where $x_{i}$ denotes the $i^{\text {th }}$ bit of $x$. In other words, the first argument of $g$ is a $k$ bit string $x$, and the second argument selects a bit of $x$ or its negation. So here $X=\{-1,+1\}^{k}, Y=[k] \times\{-1,+1\}$ and the intermediate set $E$ is $\{-1,+1\}$. With this $g$, Sherstov shows that the approximate polynomial degree of $f$ is a lower bound on the quantum communication complexity of $f \circ g^{n}$, for any function $f$. Though seemingly quite special, pattern matrices have proven to be an extremely useful concept. First, they give a simple proof of Razborov's tight lower bounds for $f(x \wedge y)$ for symmetric $f$. Second, they have also found many other applications in unbounded-error communication complexity [She08b,RS08] and have been successfully extended to multiparty communication complexity [Cha07,LS09a,CA08,BHN09].

A key step in both the works of Sherstov and Shi-Zhu is to bound the spectral norm of a sum of matrices $\left\|\sum_{i} B_{i}\right\|$. This is the major step where these works differ. Shi-Zhu apply the triangle inequality to bound this as $\left\|\sum_{i} B_{i}\right\| \leq \sum_{i}\left\|B_{i}\right\|$. On the other hand, Sherstov observes that in the case of pattern matrices the terms of this sum are mutually orthogonal, i.e. $B_{i}^{\dagger} B_{j}=B_{i} B_{j}^{\dagger}=0$ for all $i \neq j$. In this case, one has a stronger bound on the spectral norm $\left\|\sum_{i} B_{i}\right\|=\max _{i}\left\|B_{i}\right\|$.

In this paper, we extend both of the frameworks of Sherstov and Shi-Zhu. In the case of Shi-Zhu, we are able to reprove their theorem with the usual notion of discrepancy instead of the somewhat awkward spectral discrepancy they use. The main observation we make is that as all Shi-Zhu use in this step is the triangle inequality, we can repeat the argument with any norm here, including discrepancy itself.

In the case of pattern matrices, special properties of the spectral norm are used, namely the fact about the spectral norm of a sum of orthogonal matrices. We step back to see what 
key features of a pattern matrix lead to this orthogonality property. We begin with the Boolean case, that is, where the intermediate set $E$ is taken to be $\{-1,+1\}$. In this case, a crucial concept is the notion of a strongly balanced function. We say that $g: X \times Y \rightarrow\{-1,+1\}$ is strongly balanced if in the sign matrix $M_{g}[x, y]=g(x, y)$ all rows and all columns sum to zero. We show that whenever the inner function $g$ is strongly balanced, the key orthogonality condition holds; this implies that whenever $g$ is strongly balanced and the communication matrix of $g$ has rank larger than one, the approximate degree of the outer function $f$ is a lower bound on the quantum communication complexity of $f \circ g^{n}$.

The requirement that the communication matrix of $g$ has rank larger than one is necessary for such a statement. For example, when $g(x, y)=\oplus(x, y)$ is the XOR function on one bit, then the communication complexity of PARITY $\circ g^{n}$ is constant, while PARITY has linear approximate polynomial degree. It turns out that when $g$ is rank-one, the appropriate measure of the complexity of $f \circ g^{n}$ is no longer the approximate degree of $f$, but the minimum $\ell_{1}$ norm of Fourier coefficients of a function entrywise close to $f$; see the survey [LS09b] for a description of this case.

We also consider the general case where the intermediate set is any group $G$. That is, we consider functions $F(x, y)=f(g(x, y))$, where $g: X \times Y \rightarrow G$ for a group $G$ and $f$ : $G \rightarrow\{-1,+1\}$ is a class function on $G$. The case $E=\{-1,+1\}$ discussed above corresponds to taking the group $G=\mathbb{Z}_{2}^{n}$. When $G$ is a general Abelian group, the key orthogonality condition requires more than that the matrix $M_{g}[x, y]=g(x, y)$ is strongly balanced; still, it admits a nice characterization in terms of group invariance. A multiset $T \in G \times G$ is said to be $G$-invariant if $(s, s) T=T$ for all $s \in G$. The orthogonality condition will hold if and only if all pairs of rows and all pairs of columns of $M_{g}$ (when viewed as multisets) are $G$ - invariant. One can generalize the results discussed above to this general setting with appropriate modifications. In the case that $G=\mathbb{Z}_{2}^{n}$, the $G$-invariant condition degenerates to the strongly balanced requirement of $M_{g}$.

\section{Preliminaries}

All logarithms are base two. For a complex number $z=a+i b$ we let $\bar{z}=a-i b$ denote the complex conjugate of $z$ and $|z|=\sqrt{a^{2}+b^{2}}$ and $\operatorname{Re}(z)=a$.

\subsection{Complexity measures}

We will make use of several complexity measures of functions and matrices. Let $f:\{-1,+1\}^{n} \rightarrow$ $\{-1,+1\}$ be a function. For $T \subseteq\{0,1\}^{n}$, the Fourier coefficient of $f$ corresponding to the character $\chi_{T}$ is $\hat{f}_{T}=\frac{1}{2^{n}} \sum_{x} f(x) \chi_{T}(x)=\frac{1}{2^{n}} \sum_{x} f(x) \prod_{i \in T} x_{i}$. The degree of $f$ as a polynomial, denoted $\operatorname{deg}(f)$, is the size of a largest set $T$ for which $\hat{f}_{T} \neq 0$.

We will need some notations for matrices. We reserve $J$ for the all ones matrix, whose size will be determined by the context. For a matrix $A$ let $A^{\dagger}$ denote the conjugate transpose of $A$. We use $A \bullet B$ for the entrywise product of $A, B$, and $A \otimes B$ for the tensor product. If $A$ is an $m$-by- $n$ matrix then we say that $\operatorname{size}(A)=m n$. We use $\langle A, B\rangle=\operatorname{Tr}\left(A B^{\dagger}\right)$ for the inner product of $A$ and $B$.

Let $\|A\|_{1}$ be the $\ell_{1}$ norm of $A$, i.e. sum of the absolute values of entries of $A$, and $\|A\|_{\infty}$ the $\ell_{\infty}$ norm. For a positive semidefinite matrix $M$ let $\lambda_{1}(M) \geq \cdots \geq \lambda_{n}(M) \geq 0$ be the eigenvalues of $M$. We define the $i^{t h}$ singular value of $A$, denoted $\sigma_{i}(A)$, as $\sigma_{i}(A)=\sqrt{\lambda_{i}\left(A A^{\dagger}\right)}$. The rank of $A$, denoted $\operatorname{rk}(A)$ is the number of nonzero singular values of $A$. We will use several matrix norms. The spectral or operator norm is the largest singular value $\|A\|=$ $\sigma_{1}(A)$, the trace norm is the summation of all singular values $\|A\|_{t r}=\sum_{i} \sigma_{i}(A)$, and the Frobenius norm is the $\ell_{2}$ norm of the singular values $\|A\|_{F}=\sqrt{\sum_{i} \sigma_{i}(A)^{2}}$. 
When $A B^{\dagger}=A^{\dagger} B=0$ we will say that $A, B$ are orthogonal. Please note the difference with the common use of this term, which usually means $\langle A, B\rangle=0$. The following facts are easily seen.

Fact 1 Let $A, B$ be two matrices of the same dimensions and suppose that $A B^{\dagger}=A^{\dagger} B=0$. Then

$$
\operatorname{rk}(A+B)=\operatorname{rk}(A)+\operatorname{rk}(B),\|A+B\|_{t r}=\|A\|_{t r}+\|B\|_{t r},\|A+B\|=\max \{\|A\|,\|B\|\} .
$$

Another norm we will use is the $\gamma_{2}$ norm, introduced to complexity theory in [LMSS07], and familiar in matrix analysis as the Schur product operator norm. The $\gamma_{2}$ norm can be viewed as a weighted version of the trace norm.

\section{Definition 1.}

$$
\gamma_{2}(A)=\max _{u, v:\|u\|=\|v\|=1}\left\|A \bullet u v^{\dagger}\right\|_{t r}
$$

Here $A \bullet B$ denotes the entrywise product of $A$ and $B$. It is clear from this definition that $\gamma_{2}(A) \geq\|A\|_{t r} / \sqrt{m n}$ for a $m$-by- $n$ matrix $A$.

For a norm $\Phi$, the dual norm $\Phi^{*}$ is defined as $\Phi^{*}(v)=\max _{u: \Phi(u) \leq 1}|\langle u, v\rangle|$. For example, the $\ell_{\infty}$ norm is dual to the $\ell_{1}$ norm, and the spectral norm is dual to the trace norm.

The norm $\gamma_{2}^{*}$, dual to the $\gamma_{2}$ norm, looks as follows.

\section{Definition 2.}

$$
\gamma_{2}^{*}(A)=\max _{\substack{u_{i}, v_{j} \\\left\|u_{i}\right\|=\left\|v_{j}\right\|=1}} \sum_{i, j} A[i, j]\left\langle u_{i}, v_{j}\right\rangle
$$

Another complexity measure we will make use of is discrepancy

Definition 3. Let $A$ be an $m$-by-n sign matrix and let $P$ be a probability distribution on the entries of $A$. The discrepancy of $A$ with respect to $P$, denoted $\operatorname{disc}_{P}(A)$, is defined as

$$
\operatorname{disc}_{P}(A)=\max _{\substack{x \in\{0,1\}^{m} \\ y \in\{0,1\}^{n}}}\left|x^{\dagger} A \bullet P y\right|
$$

We will write $\operatorname{disc}_{U}(A)$ for the special case where $P$ is the uniform distribution. It is easy to see from this definition that $\operatorname{disc}_{U}(A) \leq \frac{\|A\|}{\sqrt{\operatorname{size}(A)}}$. Shaltiel [Sha03] has shown the deeper result that this bound is in fact polynomially tight:

Theorem 2 (Shaltiel). Let $A$ be a sign matrix. Then

$$
\frac{1}{108}\left(\frac{\|A\|}{\sqrt{\operatorname{size}(A)}}\right)^{3} \leq \operatorname{disc}_{U}(A) .
$$

Discrepancy and the $\gamma_{2}^{*}$ norm are very closely related. Linial and Shraibman [LS09c] observed that Grothendieck's inequality gives the following.

Theorem 3 (Linial-Shraibman). For any sign matrix $A$ and probability distribution $P$

$$
\operatorname{disc}_{P}(A) \leq \gamma_{2}^{*}(A \bullet P) \leq K_{G} \operatorname{disc}_{P}(A)
$$

where $1.67 \ldots \leq K_{G} \leq 1.78 \ldots$ is Grothendieck's constant. 
Approximate measures We will also use approximate versions of these complexity measures which come in handy when working with bounded-error models. Say that a function $g$ gives an $\epsilon$-approximation to $f$ if $|f(x)-g(x)| \leq \epsilon$ for all $x \in\{-1,+1\}^{n}$. The $\epsilon$-approximate polynomial degree of $f$, denoted $\operatorname{deg}_{\epsilon}(f)$, is the minimum degree of a function $g$ which gives an $\epsilon$-approximation to $f$.

We will similarly look at the $\epsilon$-approximate version of the trace and $\gamma_{2}$ norms. We give the general definition with respect to any norm.

Definition 4 (approximation norm). Let $\Phi: \mathbb{R}^{n} \rightarrow \mathbb{R}$ be an arbitrary norm. Let $v \in \mathbb{R}^{n}$ be a sign vector. For $0 \leq \epsilon<1$ we define the approximation norm $\Phi^{\epsilon}$ as

$$
\Phi^{\epsilon}(v)=\min _{\substack{u \\\|v-u\|_{\infty} \leq \epsilon}} \Phi(u) .
$$

Notice that an approximation norm $\Phi^{\epsilon}$ is not itself a norm - we have only defined it for sign vectors, and it will in general not satisfy the triangle inequality.

As a norm is a convex function, using the separating hyperplane theorem one can quite generally give the following equivalent dual formulation of an approximation norm.

Proposition 1. Let $v \in \mathbb{R}^{n}$ be a sign vector, and $0 \leq \epsilon<1$

$$
\Phi^{\epsilon}(v)=\max _{u} \frac{|\langle v, u\rangle|-\epsilon\|u\|_{1}}{\Phi^{*}(u)}
$$

A proof of this can be found in the survey [LS09b].

\subsection{Communication complexity}

Let $X, Y, S$ be finite sets and $f: X \times Y \rightarrow S$ be a function. We will let $D(f)$ be the deterministic communication complexity of $f$, and $R_{\epsilon}(f)$ denote the randomized public coin complexity of $f$ with error probability at most $\epsilon$. We refer to the reader to [KN97] for a formal definition of these models. We will also study $Q_{\epsilon}(f)$ and $Q_{\epsilon}^{*}(f)$, the $\epsilon$-error quantum communication complexity of $f$ without and with shared entanglement, respectively. We refer the reader to [Raz03] for a nice description of these models.

For notational convenience, we will identify a function $f: X \times Y \rightarrow\{-1,+1\}$ with its sign matrix $M_{f}=[f(x, y)]_{x, y}$. Thus, for example, $\|f\|$ refers to the spectral norm of the sign matrix representation of $f$.

For all of our lower bound results we will actually lower bound the approximate trace norm or $\gamma_{2}$ norm of the function. Razborov showed that the approximate trace norm can be used to lower bound on quantum communication complexity, and Linial and Shraibman generalized this to the $\gamma_{2}$ norm.

Theorem 4 (Linial-Shraibman [LS09d]). Let $A$ be a sign matrix and $0 \leq \epsilon<1 / 2$. Then

$$
Q_{\epsilon}^{*}(A) \geq \log \left(\gamma_{2}^{2 \epsilon}(A)\right)-2 .
$$

Composed functions Before discussing lower bounds on a block composed function $f \circ g^{n}$, let us see what we expect the complexity of such a function to be. A fundamental idea going back to Nisan [Nis94] and Buhrman, Cleve, and Wigderson [BCW98], is that the complexity of $f \circ g^{n}$ can be related to the decision tree complexity, also known as query complexity, of $f$ and the communication complexity of $g$. Let DT $(f)$ be the query complexity of $f$, that is the number of queries of the form $x_{i}=$ ? needed to evaluate $f(x)$ in the worst case. Similarly, let $\mathrm{RT}_{\epsilon}(f), \mathrm{QT}_{\epsilon}(f)$ denote the randomized and quantum query complexity of $f$ respectively, with error probability at most $\epsilon$. For formal definitions of these measures and a survey of query complexity we recommend Buhrman and de Wolf [BW02]. 
Theorem 5 (Nisan [Nis94], Buhrman-Cleve-Wigderson [BCW98]). For any two Boolean functions $f:\{-1,+1\}^{n} \rightarrow\{-1,+1\}$ and $g: X \times Y \rightarrow\{-1,+1\}$,

$$
\begin{aligned}
D\left(f \circ g^{n}\right) & =O(\mathrm{DT}(f) D(g)) \\
R_{1 / 4}\left(f \circ g^{n}\right) & =O\left(\mathrm{RT}_{1 / 4}(f) R_{1 / 4}(g) \log \mathrm{RT}_{1 / 4}(f)\right) \\
Q_{1 / 4}\left(f \circ g^{n}\right) & =O\left(\mathrm{QT}_{1 / 4}(f) Q_{1 / 4}(g) \log n\right) .
\end{aligned}
$$

One advantage of working with block composed functions in light of this upper bound is that query complexity is in general better understood than communication complexity. In particular, a polynomial relationship between deterministic query complexity and degree, and randomized and quantum query complexities and approximate degree is known.

Theorem 6 ([NS94,BBC $\left.\left.{ }^{+} \mathbf{0 1}\right]\right)$. Let $f:\{0,1\}^{n} \rightarrow\{-1,+1\}$. Then

$$
\mathrm{DT}(f)=O\left(\operatorname{deg}(f)^{4}\right), \quad \mathrm{DT}(f)=O\left(\operatorname{deg}_{1 / 4}(f)^{6}\right)
$$

Using this result together with Theorem 5 gives the following corollary:

\section{Corollary 1.}

$$
D\left(f \circ g^{n}\right)=O\left(\operatorname{deg}(f)^{4} D(g)\right), \quad R_{1 / 4}\left(f \circ g^{n}\right)=O\left(\operatorname{deg}_{1 / 4}(f)^{6} R_{1 / 4}(g) \log \operatorname{deg}_{1 / 4}(f)\right)
$$

Our goal, then, in showing lower bounds on the complexity of a block composed function $f \circ g^{n}$ is to get something at least in the ballpark of this upper bound. Of course, this is not always possible - the protocol given by Theorem 5 is not always optimal. For example, when $f$ is the PARITY function on $n$ bits, and $g(x, y)=\oplus(x, y)$ this protocol just gives an upper bound of $n$ bits, when the true complexity is constant. See recent results by Zhang [Zha09] and Sherstov [She10] for discussions on the tightness of the bounds in Theorem 5.

\section{Rank of block composed functions}

We begin by analyzing the rank of a block composed function $f \circ g^{n}$ when the inner function $g$ is strongly balanced. This case will illustrate the use of the strongly balanced assumption, and is simpler to understand than the bounded-error situation treated in the next section.

Let us first formally state the definition of strongly balanced.

Definition 5 (strongly balanced). Let $A$ be a sign matrix, and $J$ be the all ones matrix of the same dimensions as $A$. We say that $A$ is balanced if $\operatorname{Tr}\left(A J^{\dagger}\right)=0$. We further say that $A$ is strongly balanced if $A J^{\dagger}=A^{\dagger} J=0$. In words, a sign matrix is strongly balanced if the sum over each row is zero, and similarly the sum over each column is zero. We will say that a two-variable Boolean function is balanced or strongly balanced if its sign matrix representation is.

Theorem 7. Let $f:\{-1,+1\}^{n} \rightarrow\{-1,+1\}$ be an arbitrary function, and let $g$ be a strongly balanced function. Then

$$
\operatorname{rk}\left(M_{f \circ g^{n}}\right)=\sum_{T \subseteq[n], \hat{f}_{T} \neq 0} \operatorname{rk}\left(M_{g}\right)^{|T|}
$$

Proof. Let us write out the sign matrix for $\chi_{T} \circ g^{n}$ explicitly. If we let $M_{g}^{0}=J$ be the all ones matrix and $M_{g}^{1}=M_{g}$, then we can nicely write the sign matrix representing $\chi_{T}\left(g\left(x^{1}, y^{1}\right), \ldots, g\left(x^{n}, y^{n}\right)\right)$ as

$$
M_{\chi_{T} \circ g^{n}}=\bigotimes_{i} M_{g}^{T[i]}
$$


where $T[i]=1$ if $i \in T$ and 0 otherwise.

We see that the condition on $g$ implies $M_{\chi_{T} \circ g^{n}} M_{\chi_{S} \circ g^{n}}^{\dagger}=0$ if $S \neq T$. Indeed,

$$
\begin{aligned}
M_{\chi_{T} \circ g^{n}} M_{\chi_{S} \circ g^{n}}^{\dagger} & =\left(\bigotimes_{i} M_{g}^{T[i]}\right)\left(\bigotimes_{i} M_{g}^{S[i]}\right)^{\dagger} \\
& =\bigotimes_{i}\left(M_{g}^{T[i]}\left(M_{g}^{S[i]}\right)^{\dagger}\right)=0 .
\end{aligned}
$$

This follows since, by the assumption $S \neq T$, there is some $i$ for which $S[i] \neq T[i]$ which means that this term is either $M_{g} J^{\dagger}=0$ or $J M_{g}^{\dagger}=0$ because $g$ is strongly balanced. The other case follows similarly.

Now that we have established this property, we can use Fact 1 to obtain

$$
\begin{aligned}
\operatorname{rk}\left(M_{f \circ g^{n}}\right) & =\operatorname{rk}\left(\sum_{T \subseteq[n]} \hat{f}_{T} \chi_{T}\left(g\left(x^{1}, y^{1}\right), \ldots, g\left(x^{n}, y^{n}\right)\right)\right) \\
& =\sum_{\substack{T \subseteq[n] \\
\hat{f}_{T} \neq 0}} \operatorname{rk}\left(M_{\chi_{T} \circ g^{n}}\right) \\
& =\sum_{\substack{T \subseteq[n] \\
\hat{f}_{T} \neq 0}} \operatorname{rk}\left(M_{g}\right)^{|T|}
\end{aligned}
$$

In the last step we used the fact that rank is multiplicative under tensor product.

Theorem 7 has the following implication for the log rank conjecture of the composed function with the assumption of the same conjecture for the inner function.

Corollary 2. Let $X, Y$ be finite sets, $g: X \times Y \rightarrow\{-1,+1\}$ be a strongly balanced function, and $M_{g}[x, y]=g(x, y)$ be the corresponding sign matrix. Let $f:\{-1,+1\}^{n} \rightarrow\{-1,+1\}$ be an arbitrary function. Assume that $\operatorname{rk}\left(M_{g}\right) \geq 2$ and further suppose that there is a constant c such that $D(g) \leq\left(\log \operatorname{rk}\left(M_{g}\right)\right)^{c}$. Then

$$
D\left(f \circ g^{n}\right)=O\left(\log \operatorname{rk}(f \circ g)^{4+c}\right) .
$$

Proof. By Corollary 1, $D\left(f \circ g^{n}\right)=O\left(\operatorname{deg}(f)^{4} D(g)\right)=O\left(\operatorname{deg}(f)^{4}\left(\log \operatorname{rk}\left(M_{g}\right)\right)^{c}\right)$. Now, it follows from Theorem 7 that $\log \operatorname{rk}(f \circ g) \geq \operatorname{deg}(f) \log \operatorname{rk}\left(M_{g}\right)$ as by definition of degree there is some $T \subseteq\{0,1\}^{n}$ with $|T|=\operatorname{deg}(f)$ and $\hat{f}_{T} \neq 0$.

In particular, this Corollary means that whenever $g$ is a strongly balanced function on a constant number of bits and $\operatorname{rk}\left(M_{g}\right)>1$, then the log rank conjecture holds for $f \circ g^{n}$. If $g$ is strongly balanced and $\operatorname{rk}\left(M_{g}\right)=1$ then, up to permutation of rows and columns, which does not change the communication complexity, $M_{g}$ is a tensor product of the XOR function with an all ones matrix. The log rank conjecture in the case $\left(f \circ \oplus^{n}\right)(x, y)=f\left(x_{1} \oplus y_{1}, \ldots, x_{n} \oplus y_{n}\right)$ remains an interesting open question. Shi and Zhang [SZ09a] have recently resolved this question when $f$ is symmetric.

\section{A bound in terms of approximate degree}

In this section, we will address the frameworks of Sherstov and Shi-Zhu. We extend both of these frameworks to give more general conditions on the inner function $g$ which still imply that the approximate degree of $f$ is a lower bound on the quantum query complexity of 
the composed function $f \circ g^{n}$. In outline, both of these frameworks follow the same plan. By Theorem 4 it suffices to lower bound the approximate $\gamma_{2}$ norm (or even approximate trace norm) of $f \circ g^{n}$. To do this, they use the dual formulation given by Proposition 1 and construct a witness matrix $B$ which has non-negligible correlation with the target function and small $\gamma_{2}^{*}$ (or spectral) norm.

A very nice way to construct this witness, used by both Sherstov and Shi-Zhu, is to use the dual polynomial of $f$. This is a polynomial $v$ which certifies that the approximate polynomial degree of $f$ is at least a certain value. More precisely, duality theory of linear programming gives the following lemma.

Lemma 1 (Sherstov [She09], Shi-Zhu [SZ09b]). Let $f:\{-1,+1\}^{n} \rightarrow\{-1,+1\}$ and let $d=\operatorname{deg}_{\epsilon}(f)$. Then there exists a function $v:\{-1,+1\}^{n} \rightarrow \mathbb{R}$ such that

1. $\left\langle v, \chi_{T}\right\rangle=0$ for every character $\chi_{T}$ with $|T|<d$.

2. $\|v\|_{1}=1$.

3. $\langle v, f\rangle \geq \epsilon$.

Items $(2),(3)$ are used to lower bound the correlation of the witness matrix with the target matrix and to upper bound the $\ell_{1}$ norm of the witness matrix. In the most difficult step, and where these works diverge, Item (1) is used to upper bound the $\gamma_{2}^{*}$ (or spectral) norm of the witness matrix.

We treat each of these frameworks separately in the next two sections.

\subsection{Sherstov's framework}

The proof of the next theorem follows the same steps as Sherstov's proof for pattern matrices (Theorem 5.1 [She09]). Our main contribution is to identify the strongly balanced condition as the key property of pattern matrices which enables the proof to work.

Theorem 8. Let $X, Y$ be finite sets, $g: X \times Y \rightarrow\{-1,+1\}$ be a strongly balanced function, and $M_{g}[x, y]=g(x, y)$ be the corresponding sign matrix. Let $f:\{-1,+1\}^{n} \rightarrow\{-1,+1\}$ be an arbitrary function. Then

$$
Q_{\epsilon}^{*}\left(f \circ g^{n}\right) \geq \operatorname{deg}_{\epsilon_{0}}(f) \log _{2}\left(\frac{\sqrt{|X||Y|}}{\left\|M_{g}\right\|}\right)-O(1) .
$$

for any $\epsilon>0$ and $\epsilon_{0}>2 \epsilon$.

In particular, this result means that the quantum and randomized complexities of $f \circ g^{n}$ are polynomially related whenever $g$ is strongly balanced and $\log \frac{\sqrt{\operatorname{size}\left(M_{g}\right)}}{\left\|M_{g}\right\|}$ is polynomially related to the randomized communication complexity of $g$. While the complexity measure of $g$ used here may look strange at first, Shaltiel [Sha03] has shown that it is closely related to the discrepancy of $g$ under the uniform distribution, as noted above in Theorem 2. This theorem strictly generalizes the case of pattern matrices, but it could still be the case that the results of Shi-Zhu can show bounds not possible with this theorem.

Proof (Proof of Theorem 8). Let $d=\operatorname{deg}_{\epsilon_{0}}(f)$ and let $v$ be a dual polynomial for $f$ with properties as in Lemma 1 . We define a witness matrix as

$$
B[x, y]=\frac{2^{n}}{\operatorname{size}\left(M_{g}\right)^{n}} v\left(g\left(x^{1}, y^{1}\right), \ldots, g\left(x^{n}, y^{n}\right)\right)
$$


Let us first lower bound the inner product $\left\langle M_{f \circ g^{n}}, B\right\rangle$. Notice that as $M_{g}$ is strongly balanced, it is in particular balanced, and so the number of ones (or minus ones) in $M_{g}$ is $\operatorname{size}\left(M_{g}\right) / 2$.

$$
\left\langle M_{f \circ g^{n}}, B\right\rangle=\frac{2^{n}}{\operatorname{size}\left(M_{g}\right)^{n}} \sum_{z \in\{-1,+1\}^{n}} f(z) v(z) \prod_{i=1}^{n}\left(\sum_{\substack{x^{i}, y^{i}: \\ g\left(x^{i}, y^{i}\right)=z_{i}}} 1\right)=\langle f, v\rangle \geq \epsilon_{0}
$$

A similar argument shows that $\|B\|_{1}=1$ as $\|v\|_{1}=1$.

Now we turn to evaluate $\|B\|$. As shown above, the strongly balanced property of $g$ implies that the matrices $\chi_{T} \circ g^{n}$ and $\chi_{S} \circ g^{n}$ are orthogonal for distinct sets $S, T \subseteq\{0,1\}^{n}$. We can thus use Fact 1 to compute as follows.

$$
\begin{aligned}
\|B\| & =\frac{2^{n}}{\operatorname{size}\left(M_{g}\right)^{n}}\left\|\sum_{T \subseteq[n]} \hat{v}_{T} M_{\chi_{T} \circ g^{n}}\right\| \\
& =\frac{2^{n}}{\operatorname{size}\left(M_{g}\right)^{n}} \max _{T}\left|\hat{v}_{T}\right|\left\|M_{\chi_{T} \circ g^{n}}\right\| \\
& =\max _{T} 2^{n}\left|\hat{v}_{T}\right| \prod_{i} \frac{\left\|M_{g}^{T[i]}\right\|}{\operatorname{size}\left(M_{g}\right)} \\
& \leq \max _{T: \hat{v}_{T} \neq 0} \prod_{i} \frac{\left\|M_{g}^{T[i]}\right\|}{\operatorname{size}\left(M_{g}\right)} \\
& =\left(\frac{\left\|M_{g}\right\|}{\sqrt{\operatorname{size}\left(M_{g}\right)}}\right)^{d}\left(\frac{1}{\operatorname{size}\left(M_{g}\right)}\right)^{n / 2}
\end{aligned}
$$

In the second to last step we have used that $\left|\hat{v}_{T}\right| \leq 1 / 2^{n}$ as $\|v\|_{1}=1$, and in the last step we have used the fact that $\|J\|=\sqrt{\operatorname{size}\left(M_{g}\right)}$.

Now putting everything together we have

$$
\frac{\left\|M_{f \circ g^{n}}\right\|_{t r}^{\epsilon_{0}}}{\sqrt{\operatorname{size}\left(M_{f \circ g^{n}}\right)}} \geq \frac{1}{12}\left(\frac{\sqrt{\operatorname{size}\left(M_{g}\right)}}{\left\|M_{g}\right\|}\right)^{d}
$$

The lower bound on quantum communication complexity now follows from Theorem 4 .

Using the theorem of Shaltiel relating discrepancy to the spectral norm Theorem 2, we get the following corollary:

Corollary 3. Let the quantities be defined as in Theorem 8.

$$
Q_{1 / 8}^{*}\left(f \circ g^{n}\right) \geq \frac{1}{3} \operatorname{deg}_{1 / 3}(f)\left(\log \left(\frac{1}{\operatorname{disc}_{U}\left(M_{g}\right)}\right)-7\right)-O(1) .
$$

Comparison to Sherstov's pattern matrix: As mentioned in [She09], Sherstov's pattern matrix method can prove quantum lower bound of $\Omega\left(\operatorname{deg}_{\epsilon}(f)\right)$ for block composed functions $f \circ g^{n}$ if the matrix $M_{g}$ contains the following $4 \times 4$ one as a submatrix:

$$
S_{4}=\left[\begin{array}{cccc}
1 & -1 & 1 & -1 \\
1 & -1 & -1 & 1 \\
-1 & 1 & 1 & -1 \\
-1 & 1 & -1 & 1
\end{array}\right]
$$

In this paper we show that the same lower bound holds as long as $M_{g}$ contains a strongly balanced submatrix or rank greater than one. Are there strongly balanced matrices not 
containing $S_{4}$ as a submatrix? It turns out that the answer is yes: we give the following $6 \times 6$ matrix as one example.

$$
S_{6}=\left[\begin{array}{cccccc}
1 & 1 & 1 & -1 & -1 & -1 \\
1 & 1 & -1 & 1 & -1 & -1 \\
1 & -1 & -1 & -1 & 1 & 1 \\
-1 & -1 & 1 & 1 & 1 & -1 \\
-1 & 1 & -1 & -1 & 1 & 1 \\
-1 & -1 & 1 & 1 & -1 & 1
\end{array}\right]
$$

\subsection{Shi-Zhu framework}

The method of Shi-Zhu does not restrict the form of the inner function $g$, but rather works for any $g$ which is sufficiently "hard." The hardness condition they require is phrased in terms of a somewhat awkward measure they term spectral discrepancy.

Definition 6 (spectral discrepancy). Let $A$ be a m-by-n sign matrix. The spectral discrepancy of $A$, denoted $\rho(A)$, is the smallest $r$ such that there is a submatrix $A^{\prime}$ of $A$ and a probability distribution $\mu$ on the entries of $A^{\prime}$ satisfying:

1. $A^{\prime}$ is balanced with respect to $\mu$, i.e. the distribution which gives equal weight to -1 entries and +1 entries of $A^{\prime}$.

2. The spectral norm of $A^{\prime} \bullet \mu$ is small:

$$
\left\|A^{\prime} \bullet \mu\right\| \leq \frac{r}{\sqrt{\operatorname{size}\left(A^{\prime}\right)}}
$$

3. The entrywise absolute value of the matrix $A^{\prime} \bullet \mu$ should also have a bound on its spectral norm in terms of $r$ :

$$
\left\|\left|A^{\prime} \bullet \mu\right|\right\| \leq \frac{1+r}{\sqrt{\operatorname{size}\left(A^{\prime}\right)}}
$$

While conditions (1),(2) in the definition of spectral discrepancy are quite natural, condition (3) can be complicated to verify. Note that condition (3) will always be satisfied when $\mu$ is taken to be the uniform distribution. Using this notion of spectral discrepancy, Shi-Zhu show the following theorem.

Theorem 9 (Shi-Zhu [SZ09b]). Let $f:\{-1,+1\}^{n} \rightarrow\{-1,+1\}$, and $g: X \times Y \rightarrow$ $\{-1,+1\}$. For any $\epsilon$ and $\epsilon_{0}>2 \epsilon$,

$$
Q_{\epsilon}\left(f \circ g^{n}\right) \geq \Omega\left(\operatorname{deg}_{\epsilon_{0}}(f)\right) .
$$

provided $\rho\left(M_{g}\right) \leq \frac{\operatorname{deg}_{\epsilon_{0}}(f)}{2 e n}$. Here $e=2.718 \ldots$ is Euler's number.

Chattopadhyay [Cha08] extended the technique of Shi-Zhu to the case of multiparty communication complexity, answering an open question of Sherstov [She08a]. In doing so, he gave a more natural condition on the hardness of $g$ in terms of an upper bound on discrepancy frequently used in the multiparty setting and originally due to Babai, Nisan, and Szegedy [BNS92]. As all that is crucially needed is subadditivity, we do the argument here with $\gamma_{2}^{*}$, which is essentially equal to the discrepancy.

Theorem 10. Let $f:\{-1,+1\}^{n} \rightarrow\{-1,+1\}$, and $g: X \times Y \rightarrow\{-1,+1\}$. Fix $0<\epsilon<1 / 2$, and let $\epsilon_{0}>2 \epsilon$. Then

$$
Q_{\epsilon}^{*}\left(f \circ g^{n}\right) \geq \operatorname{deg}_{\epsilon_{0}}(f)-O(1) .
$$

provided there is a distribution $\mu$ which is balanced with respect to $g$ and for which $\gamma_{2}^{*}\left(M_{g} \bullet\right.$ $\mu) \leq \frac{\operatorname{deg}_{\epsilon_{0}}(f)}{2 e n}$. 
Proof. We again use Proposition 1, this time with the $\gamma_{2}$ norm instead of the trace norm.

$$
\gamma_{2}^{\epsilon_{0}}\left(M_{f \circ g^{n}}\right)=\max _{B} \frac{\left\langle M_{f \circ g^{n}}, B\right\rangle-\epsilon_{0}\|B\|_{1}}{\gamma_{2}^{*}(B)} .
$$

To prove a lower bound we choose a witness matrix $B$ as follows

$$
B[x, y]=2^{n} \cdot v\left(g\left(x^{1}, y^{1}\right), \ldots, g\left(x^{n}, y^{n}\right)\right) \cdot \prod_{i=1}^{n} \mu\left(x^{i}, y^{i}\right) .
$$

where $v$ witnesses that $f$ has approximate degree at least $d=\operatorname{deg}_{\epsilon_{0}}(f)$. This definition is the same as in the previous section where $\mu$ was simply the uniform distribution. As argued before, we have $\left\langle M_{f \circ g^{n}}, B\right\rangle \geq \epsilon_{0}$ and $\|B\|_{1}=1$ because $M_{g} \bullet \mu$ is balanced.

We again expand $B$ as

$$
B=2^{n} \sum_{T:|T| \geq d} \hat{v}_{T} \bigotimes_{i=1}^{n}\left(M_{g} \bullet \mu\right)^{T(i)}
$$

where $\left(M_{g} \bullet \mu\right)^{1}=M_{g} \bullet \mu$ and $\left(M_{g} \bullet \mu\right)^{0}=\mu$.

Now comes the difference with the previous proof. As we do not have special knowledge of the function $g$, we simply bound $\gamma_{2}^{*}(B)$ using the triangle inequality.

$$
\begin{aligned}
\gamma_{2}^{*}(B) & \leq 2^{n} \sum_{T:|T| \geq d}\left|\hat{v}_{T}\right| \gamma_{2}^{*}\left(\bigotimes_{i=1}^{n}\left(M_{g} \bullet \mu\right)^{T(i)}\right) \\
& =2^{n} \sum_{T:|T| \geq d}\left|\hat{v}_{T}\right| \gamma_{2}^{*}\left(M_{g} \bullet \mu\right)^{|T|} \gamma_{2}^{*}(\mu)^{n-|T|} \\
& \leq \sum_{T:|T| \geq d} \gamma_{2}^{*}\left(M_{g} \bullet \mu\right)^{|T|},
\end{aligned}
$$

where in the last step we have used that $\gamma_{2}^{*}(\mu) \leq 1$ as $\mu$ is a probability distribution and that $\left|\hat{v}_{T}\right| \leq 2^{-n}$. In the second step (equality) we used the fact that $\gamma_{2}^{*}$ is multiplicative with respect to tensor product, a property proved in [LSŠ08]. We continue with simple arithmetic:

$$
\begin{aligned}
\gamma_{2}^{*}(B) & \leq \sum_{i=d}^{n}\left(\begin{array}{c}
n \\
i
\end{array}\right) \gamma_{2}^{*}\left(M_{g} \bullet \mu\right)^{i} \\
& \leq \sum_{i=d}^{n}\left(\frac{e n \gamma_{2}^{*}\left(M_{g} \bullet \mu\right)}{d}\right)^{i} \\
& \leq 2^{-d}
\end{aligned}
$$

provided that $\gamma_{2}^{*}\left(M_{g} \bullet \mu\right) \leq \frac{d}{2 e n}$.

\section{A general framework for functions composed through a group}

In this section we begin the study of more general function composition through a group $G$. In this case the outer function $f: G \rightarrow\{-1,+1\}$ is a class function, i.e. invariant on conjugacy classes, and the inner function $g: X \times Y \rightarrow G$ has range $G$. We define the composed function as $F(x, y)=f(g(x, y))$. In previous sections of the paper we have just dealt with the case $G=\mathbb{Z}_{2}^{n}$. 
Let us recall the basic idea of the proof of Theorem 8. To prove a lower bound on the quantum communication complexity for a composed function $f \circ g$, we constructed a witness matrix $B$ which had non-negligible correlation with $f \circ g$ and small spectral norm. To do this, following the work of Sherstov and Shi-Zhu [She09,SZ09b], we considered the dual polynomial $p$ of $f$ using LP duality. The dual polynomial has two important properties, first that $p$ has non-negligible correlation with $f$ and second that $p$ has no support on low degree polynomials. We can then use the first property to show that the composed function $p \circ g$ will give non-negligible inner product with $f \circ g$ and the second to upper bound the spectral norm of $p \circ g$. The second of these tasks is the more difficult. In the case of $G=\{-1,+1\}^{n}$, the degree of a character $\chi_{T}$ is a natural measure of how "hard" the character is - the larger $T$ is, the smaller the spectral norm of $\chi_{T} \circ g$ will be. In the general group case, however, it is less clear what the corresponding "hard" and "easy" characters should be. In Section 5.1, we will show that this framework actually works for an arbitrary partition of the basis functions into Easy and Hard. That is for any arbitrary partition of the basis functions into Easy and Hard sets we can follow the plan outlined above and look for a function with support on the Hard set which has non-negligible correlation with $f$.

In carrying out this plan, one is still left with upper bounding $\left\|M_{p \circ g}\right\|$. Here, as in the Boolean case, it as again very convenient to have an orthogonality condition which can greatly simplify the computation of $\left\|M_{p \circ g}\right\|$ and give good bounds. In the Boolean case we have shown that $M_{g}$ being strongly balanced implies this key orthogonality condition. In Section 5.2 and 5.3, we will show that for the general group, the condition is not only about each row and column of matrix $M_{g}$, but all pairs of rows and pairs of columns. In the Abelian group case, this reduces to a nice group invariance condition.

Even after applying the orthogonality condition to use the maximum bound instead of the triangle inequality for $\left\|M_{p \circ g}\right\|$, the remaining term $\left\|M_{\chi_{i} \circ g}\right\|$ (where $\chi_{i}$ is a "hard" character) is still not easy to upper bound. For block composed functions, fortunately, the tensor structure makes it feasible to compute. Section 5.4 gives a generalized version of Theorem 8 .

\subsection{General framework}

For a multiset $T, x \in T$ means $x$ running over $T$. Thus $T=\{a(s): s \in S\}$ means the multiset formed by collecting $a(s)$ with $s$ running over $S$.

For a set $S$, denote by $L_{\mathbb{C}}(S)$ the $|S|$-dimensional vector space over the field $\mathbb{C}$ (of complex numbers) consisting of all linear functions from $S$ to $\mathbb{C}$, endowed with inner product $\langle\psi, \phi\rangle=$

$\frac{1}{|S|} \sum_{s \in S} \psi(s) \overline{\phi(s)}$. The distance of a function $f \in L_{\mathbb{C}}(S)$ to a subspace $\Phi$ of $L_{\mathbb{C}}(S)$, denoted by $d(f, \Phi)$, is defined as $\min \left\{\delta:\left\|f^{\prime}-f\right\|_{\infty} \leq \delta, f^{\prime} \in \Phi\right\}$, i.e. the magnitude of the least entrywise perturbation to turn $f$ into $\Phi$.

In the above setting, Theorem 8 generalizes to the following.

Theorem 11. Consider a sign matrix $A=[f(g(x, y))]_{x, y}$ where $g: X \times Y \rightarrow S$ for a set $S$, and $f: S \rightarrow\{-1,+1\}$. Suppose that we can find an orthogonal basis functions $\Psi=\left\{\psi_{i}: i \in\right.$ $[|S|]\}$ for $L_{\mathbb{C}}(S)$. For any hardness partition $\Psi=\Psi_{H a r d} \uplus \Psi_{\text {Easy }}$, let $\delta=d\left(f, \operatorname{span}\left(\Psi_{\text {Easy }}\right)\right)$. If

1. (regularity) The multiset $\{g(x, y): x \in X, y \in Y\}$ is a multiple of $S$, i.e. $S$ repeated for some number of times.

2. (orthogonality) for all $x, x^{\prime}, y, y^{\prime}$ and all distinct $\psi_{i}, \psi_{j} \in \Psi_{H a r d}$,

$$
\sum_{y} \psi_{i}(g(x, y)) \overline{\psi_{j}\left(g\left(x^{\prime}, y\right)\right)}=\sum_{x} \psi_{i}\left(g(x, y) \overline{\psi_{j}\left(g\left(x, y^{\prime}\right)\right)}=0\right.
$$

then

$$
Q_{\epsilon}(A) \geq \log _{2} \frac{\sqrt{M N} \cdot(\delta-2 \epsilon)}{\max _{\psi_{i} \in \Psi_{H a r d}}\left(\max _{g}\left|\psi_{i}(g)\right| \cdot\left\|\left[\psi_{i}(g(x, y))\right]_{x, y}\right\|\right)}-O(1)
$$


Using the idea of finding a certificate of the high approximate degree by duality [She09,SZ09b], we have the following fact analogous to Lemma 1.

Lemma 2. For a function $f: S \rightarrow \mathbb{C}$ and a subspace $\Phi$ of $L_{\mathbb{C}}(S)$, if $d(f, \operatorname{span}(\Phi))=\delta$, then there exists a function $h$ s.t.

$$
\begin{aligned}
& \hat{h}_{i}=0, \forall \psi_{i} \in \Phi \\
& \sum_{g \in G}|h(g)| \leq 2, \\
& \left|\sum_{g \in G} f(g) \overline{h(g)}\right|>\delta
\end{aligned}
$$

Using the lemma, we can prove the Theorem 11.

Proof. (of Theorem 11) By the regularity property, we know that when $(x, y)$ runs over $X \times Y$, $g(x, y)$ runs over $S$ exactly $K$ times where $K=M N /|G|$. Consider $B=\frac{1}{K}[h(g(x, y))]_{x, y}, h$ obtained by Lemma 2; we want to apply Proposition 1 and Theorem 4 by using this $B$. First,

$$
\|B\|_{1}=\frac{1}{K} \sum_{x, y}|h(g(x, y))|=\sum_{g \in G}|h(g)| \leq 1 .
$$

Also,

$$
\begin{aligned}
|\langle A, B\rangle| & =\frac{1}{K}\left|\sum_{x, y} f(g(x, y)) \overline{h(g(x, y))}\right| \\
& =\left|\sum_{g \in G} f(g) \overline{h(g)}\right|>\delta
\end{aligned}
$$

Now we need to compute

$$
\|B\|=\frac{1}{K}\left\|\left[\sum_{\chi_{i} \in \text { Hard }} \hat{h}_{i} \chi_{i}(g(x, y))\right]_{x, y}\right\| .
$$

Note that

$$
\left[\psi_{i}(g(x, y))\right]_{x, y}^{\dagger}\left[\psi_{j}(g(x, y))\right]_{x, y}=\left[\sum_{x} \overline{\psi_{i}(g(x, y))} \psi_{j}\left(g\left(x, y^{\prime}\right)\right)\right]_{y, y^{\prime}}
$$

and

$$
\left[\psi_{i}(g(x, y))\right]_{x, y}\left[\psi_{j}(g(x, y))\right]_{x, y}^{\dagger}=\left[\sum_{y} \psi_{i}(g(x, y)) \overline{\psi_{j}\left(g\left(x^{\prime}, y\right)\right)}\right]_{x, x^{\prime}} .
$$

Thus the orthogonality condition implies that

$$
\left[\psi_{i}(g(x, y))\right]_{x, y}^{\dagger}\left[\psi_{j}(g(x, y))\right]_{x, y}=\left[\psi_{i}(g(x, y))\right]_{x, y}\left[\psi_{j}(g(x, y))\right]_{x, y}^{\dagger}=0
$$

for all $i \neq j$. Now as in [She09], we can use the max bound

$$
\begin{aligned}
\|B\| & =\frac{1}{K} \max _{i: \psi_{i} \in \Psi_{\text {Hard }}}\left\|\hat{h}_{i}\left[\psi_{i}(x, y)\right]_{x, y}\right\| \\
& \leq \frac{1}{K} \max _{i: \psi_{i} \in \text { Hard }}\left|\hat{h}_{i}\right| \max _{\psi_{i} \in \text { Hard }}\left\|\left[\psi_{i}(x, y)\right]_{x, y}\right\| \\
& \leq \frac{1}{K|G|} \max _{\psi_{i} \in \text { Hard }}\left(\max _{g}\left|\psi_{i}(g)\right| \cdot\left\|\left[\psi_{i}(x, y)\right]_{x, y}\right\|\right) .
\end{aligned}
$$

where the last inequality is due to Eq. (5) and Eq. (4). Finally note $K|G|=M N$ to complete the proof. 
In the Boolean block composed function case, the regularity condition reduces to the matrix $[g(x, y)]$ being balanced, and later we will prove that the orthogonality condition reduces to the strongly balanced property. From this theorem we can see that the way to partition $\Psi$ into $\Psi_{E a s y}$ and $\Psi_{H a r d}$ does not really matter for the lower bound proof passing through. However, the partition does play a role when we later bound the spectral norm in the denominator.

\subsection{Functions with group symmetry}

For a general finite group $G$, two elements $s$ and $t$ are conjugate, denoted by $s \sim t$, if there exists an element $r \in G$ s.t. $r s r^{-1}=t$. Define $H$ as the set of all class functions, i.e. functions $f$ s.t. $f(s)=f(t)$ if $s \sim t$. Then $H$ is an $h$-dimensional subspace of $L_{\mathbb{C}}(G)$, where $h$ is the number of conjugacy classes. The irreducible characters $\left\{\chi_{i}: i \in[h]\right\}$ form an orthogonal basis of $H$. For a class function $f$ and irreducible characters $\chi_{i}$, denote by $\hat{f}_{i}$ the coefficient of $\chi_{i}$ in expansion of $f$ according to $\chi_{i}$ 's, i.e. $\hat{f}_{i}=\left\langle\chi_{i}, f\right\rangle=\frac{1}{|G|} \sum_{g \in G} \chi_{i}(g) \overline{f(g)}$. An easy fact is that for any $i$, we have

$$
\left|\hat{f}_{i}\right|=\frac{1}{|G|}\left|\sum_{g \in G} \chi_{i}(g) \overline{f(g)}\right| \leq \frac{1}{|G|} \sum_{g \in G}|f(g)|\left|\chi_{i}(g)\right| \leq\left(\frac{1}{|G|} \sum_{g \in G}|f(g)|\right) \cdot \max _{g}\left|\chi_{i}(g)\right| .
$$

If $G$ is Abelian, then it always has $\left|\chi_{i}(g)\right|=1$, thus $\max _{i}\left|\hat{f}_{i}\right| \leq \frac{1}{|G|} \sum_{g \in G}|f(g)|$. For general groups, we have $\left|\chi_{i}(g)\right| \leq \operatorname{deg}\left(\chi_{i}\right)$, where $\operatorname{deg}\left(\chi_{i}\right)$ is the degree of $\chi_{i}$, namely the dimension of the associated vector space.

In this section we consider the setting that $S$ is a finite group $G$. The goal is to exploit properties of group characters to give better form of the lower bound. In particular, we hope to see when the second condition holds and what the matrix operator norm $\left.\|\left[\psi_{(} g(x, y)\right)\right]_{x, y} \|$ is in this setting.

The standard orthogonality of irreducible characters says that $\sum_{s \in G} \chi_{i}(s) \overline{\chi_{j}(s)}=0$. The second condition in Theorem 11 is concerned with a more general case: For a multiset $T$ with elements in $G \times G$, we need

$$
\sum_{(s, t) \in T} \chi_{i}(s) \overline{\chi_{j}(t)}=0, \quad \forall i \neq j
$$

The standard orthogonality relation corresponds to the special that $T=\{(s, s): s \in G\}$. We hope to have a characterization of a multiset $T$ to make Eq. (6) hold.

We may think of the a multiset $T$ with elements in set $S$ as a function on $S$, with the value on $s \in S$ being the multiplicity of $s$ in $T$. Since characters are class functions, for each pair $\left(C_{k}, C_{l}\right)$ of conjugacy classes, only the value $\sum_{g_{1} \in C_{k}, t \in C_{l}} T\left(g_{1}, t\right)$ matters for the sake of Eq. (6). We thus make $T$ a class function by taking average within each class pair $\left(C_{k}, C_{l}\right)$. That is, define a new function $T^{\prime}$ as

$$
T^{\prime}(s, t)=\sum_{s \in C_{k}, t \in C_{l}} T(s, t) /\left(\left|C_{k}\right|\left|C_{l}\right|\right), \forall s \in C_{k}, \forall t \in C_{l} .
$$

Proposition 2. For a finite group $G$ and a multiset $T$ with elements in $G \times G$, the following three statements are equivalent:

1. $\sum_{(s, t) \in T} \chi_{i}(s) \overline{\chi_{j}(t)}=0, \forall i \neq j$

2. $T^{\prime}$, as a function, is in $\operatorname{span}\left\{\chi_{i} \otimes \overline{\chi_{i}}: i \in[h]\right\}$

3. $\left[T^{\prime}(s, t)\right]_{s, t}=C^{\dagger} D C$ where $D$ is a diagonal matrix and $C=\left[\chi_{i}(s)\right]_{i, s}$. That is, $T^{\prime}$, as a matrix, is normal and diagonalized exactly by the irreducible characters. 
Proof. Let $H_{2}$ be the subspace consisting functions $f: G \times G \rightarrow \mathbb{C}$ s.t. $f(s, t)=f\left(s^{\prime}, t^{\prime}\right)$ if $s \sim s^{\prime}, t \sim t^{\prime}$. Note that for direct product group $G \times G,\left\{\chi_{i} \otimes \overline{\chi_{j}}: i, j\right\}$ form an orthogonal basis of $H_{2}$ :

$$
\sum_{s, t \in G} \chi_{i}(s) \overline{\chi_{j}(t)} \overline{\chi_{i^{\prime}}(s) \overline{\chi_{j^{\prime}}(t)}}=\left(\sum_{s \in G} \chi_{i}(s) \overline{\chi_{i^{\prime}}(s)}\right)\left(\sum_{t \in G} \overline{\chi_{j}(t)} \chi_{j^{\prime}}(t)\right)=0
$$

unless $i=i^{\prime}$ and $j=j^{\prime}$. Note that by viewing $T$ as a function from $G \times G$ to $\mathbb{C}$, the Eq. (6) and the definition of $T^{\prime}$ imply that

$$
\left\langle\chi_{i} \otimes \overline{\chi_{j}}, T\right\rangle=0, \forall i \neq j
$$

Thus the first two statements are equivalent.

Note that

$$
T^{\prime} \in \operatorname{span}\left\{\chi_{i} \otimes \overline{\chi_{i}}: i \in[h]\right\} \quad \Leftrightarrow \quad T^{\prime}(s, t)=\sum_{i} \alpha_{i} \chi_{i}(s) \overline{\chi_{i}(t)} \quad \text { for some } \alpha_{i}{ }^{\prime} \mathrm{s}
$$

Denote by $C_{h \times|G|}=\left[\chi_{i}(g)\right]_{i, g}$ the matrix of the character table. Then observe that the summation in the last equality is nothing but the $(s, t)$ entry of the matrix $C^{\dagger} \operatorname{diag}\left(\alpha_{1}, \cdots, \alpha_{h}\right) C$. Therefore the equivalence of the second and third statements follows.

\subsection{Abelian group}

When $G$ is Abelian, we have further properties to use. The first one is that $\left|\chi_{i}(g)\right|=1$ for all $i$. The second one is that the irreducible characters are homomorphisms of $G$; that is, $\chi_{i}(s t)=\chi_{i}(s) \chi_{i}(t)$. This gives a clean characterization of the orthogonality condition by group invariance. For a multiset $T$, denote by $s T$ another multiset obtained by collecting all st where $t$ runs over $T$. A multiset $T$ with elements in $G \times G$ is $G$ invariant if it satisfies $(g, g) T=T$ for all $g \in G$. We can also call a function $T: G \times G \rightarrow \mathbb{C} G$ invariant if $T(s, t)=T(r s, r t)$ for all $r, s, t \in G$. The overloading of the name is consistent when we view a multiset $T$ as a function (counting the multiplicity of elements).

Proposition 3. For a finite Abelian group $G$ and a multiset $T$ with elements in $G \times G$,

$$
T \text { is } G \text { invariant } \Leftrightarrow \sum_{(s, t) \in T} \chi_{i}(s) \overline{\chi_{j}(t)}=0, \quad \forall i \neq j .
$$

Proof. $\Rightarrow$ : Since $T$ is $G$ invariant, $T=(r, r) T$ and thus,

$$
\begin{aligned}
\sum_{(s, t) \in T} \chi_{i}(s) \overline{\chi_{j}(t)} & =\sum_{(s, t) \in(r, r) T} \chi_{i}(s) \overline{\chi_{j}(t)} \\
& =\sum_{\left(s^{\prime}, t^{\prime}\right) \in T} \chi_{i}\left(r s^{\prime}\right) \overline{\chi_{j}\left(r t^{\prime}\right)}
\end{aligned}
$$

Now using the fact that irreducible characters of Abelian groups are homomorphisms, we have

$$
\begin{aligned}
\sum_{\left(s^{\prime}, t^{\prime}\right) \in T} \chi_{i}\left(r s^{\prime}\right) \overline{\chi_{j}\left(r t^{\prime}\right)} & =\sum_{\left(s^{\prime}, t^{\prime}\right) \in T} \chi_{i}(r) \chi_{i}\left(s^{\prime}\right) \overline{\chi_{j}(r) \chi_{j}\left(t^{\prime}\right)} \\
& =\chi_{i}(r) \overline{\chi_{j}(r)}\left(\sum_{\left(s^{\prime}, t^{\prime}\right) \in T} \chi_{i}\left(s^{\prime}\right) \overline{\chi_{j}\left(t^{\prime}\right)}\right)
\end{aligned}
$$


But note that this holds for any $r \in G$, thus also for the average of them. That is,

$$
\sum_{(s, t) \in T} \chi_{i}(s) \overline{\chi_{j}(t)}=\frac{1}{|G|}\left(\sum_{r \in G} \chi_{i}(r) \overline{\chi_{j}(r)}\right)\left(\sum_{\left(s^{\prime}, t^{\prime}\right) \in T} \chi_{i}\left(s^{\prime}\right) \overline{\chi_{j}\left(t^{\prime}\right)}\right)=0,
$$

by the standard orthogonality property of different irreducible characters.

$\Leftarrow$ : Since $\sum_{(s, t) \in T} \chi_{i}(s) \overline{\chi_{j}(t)}=0, \forall i \neq j$, we know that $T$ as a function is in $\operatorname{span}\left\{\chi_{i} \otimes \overline{\chi_{i}}\right.$ : $i$ \}. Note that any linear combination of $G$ invariant functions is also $G$ invariant. Thus it remains to check that each basis $\chi_{i} \otimes \overline{\chi_{i}}$ is $G$ invariant, which is easy to see:

$$
\chi_{i}(r s) \overline{\chi_{i}(r t)}=\chi_{i}(r) \chi_{i}(s) \overline{\chi_{i}(r) \chi_{i}(t)}=\chi_{i}(s) \overline{\chi_{i}(t)}
$$

This finishes the proof.

Another nice property of Abelian groups is that the orthogonality condition condition implies the regularity one.

Proposition 4. For an Abelian group $G$, if either $T^{y, y}$ is $G$ invariant for all $y$ or $S^{x, x}$ is $G$ invariant for all $x$, then $G \mid\{g(x, y): x \in X, y \in Y\}$.

Proof. Note that $T^{y, y}(s, s)=|\{x: g(x, y)=s\}|$, thus $T^{y, y}$ being $G$ invariant implies that $|\{x: g(x, y)=s\}|=|\{x: g(x, y)=t\}|$ for all $s, t \in G$. Thus the column $y$ in matrix $[g(x, y)]_{x, y}$, when viewed as a multiset, is equal to $G$ repeated $|Y| /|G|$ times. Therefore the whole multiset $\{g(x, y): x \in X, y \in Y\}$ is a multiple of $G$ as well.

What we finally get for Abelian groups is the following.

Corollary 4. For a sign matrix $A=[f(g(x, y))]_{x, y}$ and an Abelian group $G$, if $d\left(f\right.$, span $\left.\left(C h_{E a s y}\right)\right)=$ $\Omega(1)$, and the multisets $S^{x, x^{\prime}}=\left\{\left(g(x, y), g\left(x^{\prime}, y\right)\right): y \in Y\right\}$ and $T^{y, y^{\prime}}=\left\{\left(g(x, y), g\left(x, y^{\prime}\right)\right)\right.$ : $x \in X\}$ are $G$ invariant for any $\left(x, x^{\prime}\right)$ and any $\left(y, y^{\prime}\right)$, then

$$
Q(A) \geq \log _{2} \frac{\sqrt{M N}}{\max _{i \in \text { Hard }}\left\|\left[\chi_{i}(g(x, y))\right]_{x, y}\right\|}-O(1) .
$$

\subsection{Block composed functions}

We now consider a special class of functions $g$ : block composed functions. Suppose the group $G$ is a product group $G=G_{1} \times \cdots \times G_{t}$, and $g(x, y)=\left(g_{1}\left(x^{1}, y^{1}\right), \cdots, g_{t}\left(x^{t}, y^{t}\right)\right)$ where $x=$ $\left(x^{1}, \cdots, x^{t}\right)$ and $y=\left(y^{1}, \cdots, y^{t}\right)$. That is, both $x$ and $y$ are decomposed into $t$ components and the $i$-th coordinate of $g(x, y)$ only depends on the $i$-th components of $x$ and $y$. The tensor structure makes all the computation easy. Theorem 8 can be generalized to the general product group case for arbitrary groups $G_{i}$.

Definition 7. The $\epsilon$-approximate degree of a class function $f$ on product group $G_{1} \times \cdots \times G_{t}$, denoted by $d_{\epsilon}(f)$, is the minimum d s.t. $\left\|f-f^{\prime}\right\|_{\infty} \leq \epsilon$, where $f^{\prime}$ can be represented as a linear combination of irreducible characters with at most $d$ non-identity component characters.

Theorem 12. For sign matrix

$$
A=\left[f\left(g_{1}\left(x^{1}, y^{1}\right), \cdots, g_{t}\left(x^{t}, y^{t}\right)\right]_{x, y}\right.
$$

where all $g_{i}$ satisfy their orthogonality conditions, we have

$$
Q(A) \geq \min _{\left\{\chi_{i}\right\}, S} \sum_{i \in S} \log _{2} \frac{\sqrt{\operatorname{size}\left(M_{g_{i}}\right)}}{\operatorname{deg}\left(\chi_{i}\right)\left\|M_{\chi_{i} \circ g_{i}}\right\|}-O(1)
$$

where the minimum is over all $S \subseteq[n]$ with $|S|>\operatorname{deg}_{1 / 3}(f)$, and all non-identity irreducible characters $\chi_{i}$ of $G_{i}$. 
Proof. Recall that an irreducible character $\chi$ of $G$ is the tensor product of irreducible characters $\chi_{i}$ of each component group $G_{i}$. Let Hard be the set of irreducible characters $\chi$ with more than $d$ non-identity component characters. Fix a hard character $\chi$, and denote by $S$ the set of coordinates of its non-identity characters.

$$
\begin{aligned}
\|[\chi(g(x, y))]\| & =\left\|\bigotimes_{i \in[t]}\left[\chi_{i}\left(g_{i}\left(x^{i}, y^{i}\right)\right)\right]\right\| \\
& =\prod_{i \in[t]}\left\|\left[\chi_{i}\left(g_{i}\left(x^{i}, y^{i}\right)\right)\right]\right\| \\
& =\prod_{i \in S}\left\|\left[\chi_{i}\left(g_{i}\left(x^{i}, y^{i}\right)\right)\right]\right\| \times \prod_{i \notin S}\left\|J_{\left|X_{i}\right| \times\left|Y_{i}\right|}\right\| \\
& =\prod_{i \in S}\left\|M_{\chi_{i} \circ g_{i}}\right\| \times \prod_{i \notin S} \sqrt{\operatorname{size}\left(M_{g_{i}}\right)}
\end{aligned}
$$

Thus by Theorem 11 and Eq. (5), we have

$$
Q(A) \geq \log _{2} \prod_{i \in S} \frac{\sqrt{\operatorname{size}\left(M_{g_{i}}\right)}}{\operatorname{deg}\left(\chi_{i}\right) \cdot\left\|M_{\chi_{i} \circ g_{i}}\right\|}-O(1)
$$

proving the theorem.

Previous sections as well as [SZ09b] consider the case where all $g_{i}$ 's are the same and all $G_{i}$ 's are $\mathbb{Z}_{2}$. In this case, the above bound is equal to the one in Theorem 8 , and the following proposition says that the group invariance condition degenerates to the strongly balanced property.

Proposition 5. For $G=\mathbb{Z}_{2}^{\times t}$, the following two conditions for $g=\left(g_{1}, \cdots, g_{t}\right)$ are equivalent:

1. The multisets $S^{x, x^{\prime}}=\left\{\left(g(x, y), g\left(x^{\prime}, y\right)\right): y \in Y\right\}$ and $T^{y, y^{\prime}}=\left\{\left(g(x, y), g\left(x, y^{\prime}\right)\right): x \in X\right\}$ are $G$ invariant for any $\left(x, x^{\prime}\right)$ and any $\left(y, y^{\prime}\right)$,

2. Each matrix $\left[g_{i}\left(x^{i}, y^{i}\right)\right]_{x^{i}, y^{i}}$ is strongly balanced.

Proof. $1 \Rightarrow 2: S^{x, x^{\prime}}$ being $G$ invariant implies that for all $\left\{z_{i}\right\},\left\{u_{i}\right\},\left\{v_{i}\right\}$,

$$
\begin{aligned}
& \left|\left\{y: z_{i} g_{i}\left(x^{i}, y^{i}\right)=u_{i}, z_{i} g_{i}\left(x^{\prime i}, y^{i}\right)=v_{i}, \forall i\right\}\right| \\
= & \left|\left\{y: g_{i}\left(x^{i}, y^{i}\right)=u_{i}, g_{i}\left(x^{\prime i}, y^{i}\right)=v_{i}, \forall i\right\}\right| .
\end{aligned}
$$

Take $x^{\prime}=x$ and $u=v$. Now for each $i$ and each row $x^{i}$, take $z_{i}=-1$ (where the group $\mathbb{Z}_{2}$ is represented by $\{ \pm 1\})$. For all other $i^{\prime} \neq i$, take $z_{i^{\prime}}=1$. This assignment will show that

$$
\left|\left\{y: g_{i}\left(x^{i}, y^{i}\right)=-u_{i}\right\}\right|=\left|\left\{y: g_{i}\left(x^{i}, y^{i}\right)=u_{i}\right\}\right| .
$$

That is, the row $x^{i}$ in matrix $\left[g_{i}\left(x^{i}, y^{i}\right)\right]$ is balanced. Similarly we can show the balance for each column.

$2 \Rightarrow 1$ : It is enough to show that for each $i$ and each $\left\{z_{i}\right\},\left\{u_{i}\right\},\left\{v_{i}\right\}$,

$$
\begin{aligned}
& \left|\left\{y^{i}: z_{i} g_{i}\left(x^{i}, y^{i}\right)=u_{i}, z_{i} g_{i}\left(x^{i}, y^{i}\right)=v_{i}\right\}\right| \\
= & \left|\left\{y^{i}: g_{i}\left(x^{i}, y^{i}\right)=u_{i}, g_{i}\left(x^{\prime i}, y^{i}\right)=v_{i}\right\}\right| .
\end{aligned}
$$

First consider the case $x^{i}=x^{i}$. If $u_{i} \neq v_{i}$ then both numbers are 0 ; if $u_{i}=v_{i}$ then both numbers are $\left|\left\{y^{i}\right\}\right| / 2$ by the balance of row $x^{i}$. Now assume $x^{\prime i} \neq x^{i}$. Denote $a_{b b^{\prime}}=\mid\left\{y^{i}\right.$ : 
$\left.g_{i}\left(x^{i}, y^{i}\right)=b, g_{i}\left(x^{i}, y^{i}\right)=b^{\prime}\right\} \mid$, then the above requirement amounts to $a_{00}=a_{11}$ and $a_{01}=a_{10}$.

Note that we have

$$
a_{00}+a_{01}=\left|\left\{y^{i}: g_{i}\left(x^{i}, y^{i}\right)=0\right\}\right|=\left|\left\{y^{i}: g_{i}\left(x^{i}, y^{i}\right)=1\right\}\right|=a_{10}+a_{11}
$$

where the second equality is due to the balance of row $x^{i}$. And similarly we have $a_{00}+a_{10}=$ $a_{01}+a_{11}$ by balance of row $x^{i}$. Combining the two, we get $a_{00}=a_{11}$ and $a_{01}=a_{10}$ as desired.

It is worth noting that the conclusion does not hold if any group $G_{i}$ with size larger than two. We omit the counterexamples here.

\section{References}

$\left[\mathrm{BBC}^{+} 01\right]$ R. Beals, H. Buhrman, R. Cleve, M. Mosca, and R. de Wolf. Quantum lower bounds by polynomials. Journal of the ACM, 48(4):778-797, 2001. Earlier version in FOCS'98.

[BCW98] H. Buhrman, R. Cleve, and A. Wigderson. Quantum vs. classical communication and computation. In Proceedings of the 30th ACM Symposium on the Theory of Computing, pages 63-68, 1998.

[BHN09] P. Beame and D. Huynh-Ngoc. Multiparty communication complexity and threshold circuit size of $\mathrm{AC}^{0}$. In Proceedings of the 50th IEEE Symposium on Foundations of Computer Science, pages 53-62, 2009.

[BNS92] L. Babai, N. Nisan, and M. Szegedy. Multiparty protocols, pseudorandom generators for Logspace, and time-space trade-offs. Journal of Computer and System Sciences, 45:204232, 1992.

[BW02] H. Buhrman and R. de Wolf. Complexity measures and decision tree complexity: A survey. Theoretical Computer Science, 288:21-43, 2002.

[CA08] A. Chattopadhyay and A. Ada. Multiparty communication complexity of disjointness. Technical Report TR-08-002, ECCC, 2008.

[Cha07] A. Chattopadhyay. Discrepancy and the power of bottom fan-in depth-three circuits. In Proceedings of the 48th IEEE Symposium on Foundations of Computer Science, pages 449-458, 2007.

[Cha08] A. Chattopadhyay. PhD thesis, McGill University, 2008.

[KN97] E. Kushilevitz and N. Nisan. Communication Complexity. Cambridge University Press, 1997.

[LMSS07] N. Linial, S. Mendelson, G. Schechtman, and A. Shraibman. Complexity measures of sign matrices. Combinatorica, 27(4):439-463, 2007.

[LS88] L. Lovász and M. Saks. Möbius functions and communication complexity. In Proceedings of the 29th IEEE Symposium on Foundations of Computer Science, pages 81-90, 1988.

[LS09a] T. Lee and A. Shraibman. Disjointness is hard in the multiparty number-on-the-forehead model. Computational Complexity, 18(2):309-336, 2009.

[LS09b] T. Lee and A. Shraibman. Lower bounds in communication complexity. Foundations and Trends in Theoretical Computer Science, 3, 2009.

[LS09c] N. Linial and A. Shraibman. Learning complexity versus communication complexity. Combinatorics, Probability, and Computing, 18:227-245, 2009.

[LS09d] N. Linial and A. Shraibman. Lower bounds in communication complexity based on factorization norms. Random Structures and Algorithms, 34:368-394, 2009.

[LSŠ08] T. Lee, A. Shraibman, and R. Špalek. A direct product theorem for discrepancy. In Proceedings of the 23rd IEEE Conference on Computational Complexity, pages 71-80. IEEE, 2008.

[Nis94] Noam Nisan. The communication complexity of threshold gates. In In Proceedings of Combinatorics, Paul Erdos is Eighty, pages 301-315, 1994.

[NS94] N. Nisan and M. Szegedy. On the degree of Boolean functions as real polynomials. Computational Complexity, 4:301-313, 1994. 
[Raz99] R. Raz. Exponential separation of quantum and classical communication complexity. In Proceedings of the 31st ACM Symposium on the Theory of Computing, pages 358-367, 1999.

[Raz03] A. Razborov. Quantum communication complexity of symmetric predicates. Izvestiya: Mathematics, 67(1):145-159, 2003.

[RS08] A. Razborov and A. Sherstov. The sign rank of $\mathrm{AC}^{0}$. In Proceedings of the 49th IEEE Symposium on Foundations of Computer Science, pages 57-66, 2008.

[Sha03] R. Shaltiel. Towards proving strong direct product theorems. Computational Complexity, 12(1-2):1-22, 2003.

[She07] A. Sherstov. Separating $\mathrm{AC}^{0}$ from depth-2 majority circuits. In Proceedings of the 39th ACM Symposium on the Theory of Computing, pages 294-301. ACM, 2007.

[She08a] A. Sherstov. Communication lower bounds using dual polynomials. Bulletin of the EATCS, 95:59-93, 2008.

[She08b] A. Sherstov. The unbounded-error communication complexity of symmetric functions. In Proceedings of the 49th IEEE Symposium on Foundations of Computer Science, 2008.

[She09] A. Sherstov. The pattern matrix method. SIAM Journal on Computing, 2009.

[She10] A. Sherstov. On quantum-classical equivalence for composed communication problems. Quantum Information and Computation, 10(5-6):435-455, 2010.

[SZ09a] Y. Shi and Z. Zhang. Communication complexities of XOR functions. Quantum information and computation, 9(3-4):255-263, 2009.

[SZ09b] Y. Shi and Y. Zhu. Quantum communication complexity of block-composed functions. Quantum information and computation, 9(5,6):444-460, 2009.

[Yao79] A. Yao. Some complexity questions related to distributive computing. In Proceedings of the 11th ACM Symposium on the Theory of Computing, pages 209-213, 1979.

[Zha09] S. Zhang. On the tightness of the Buhrman-Cleve-Wigderson simulation. In Proceedings of the 20th International Symposium on Algorithms and Computation, pages 434-440, 2009 . 